\title{
Peer Reviewers for the Journal of the American Board of Family Medicine in 2014
}

\author{
Phillip Lupo, Jr., MLIS, Anne Victoria Neale, PhD, MPH, and
}

Marjorie A. Bowman, MD, MPA

We thank our peer reviewers for their time, expertise, and dedication to the fournal of the American Board of Family Medicine ( $A A B F M)$. The $7 A B F M$ peer reviewers serve a vital role in contributing to the field of family medicine and the scientific community. We take this opportunity to acknowledge and thank our peer reviewers for their support during the past year. In 2014, 417 individuals provided 508 peer reviews and advice regarding the suitability of articles for publication in the $7 A B F M$. Reviewers took an average of 23 days to submit a review.

\section{Top Reviewers}

We would like to recognize our top reviewers. Each of the manuscripts submitted to the $7 A B F M$

Conflict of interest: The authors are editors and staff of the 7ABFM. suitable for peer review is evaluated and scored by the editors. In the list below, peer reviewers with an asterisk next to their name are our top reviewers for 2014 based on evaluation scores plus quantity of critiques provided.

\section{Seeking New Reviewers}

We also enjoy welcoming new peer reviewers into the fold. Peer reviewing is a vital service activity that helps authors and researchers improve their work. Reviewers are always free to decline a request or to indicate periods of unavailability. If you would like to volunteer to review for the $7 A B F M$, please download and complete the Peer Reviewer Form, which can be accessed at http:// jabfm.org/site/misc/PeerReviewForm.doc; then, e-mail it along with your curriculum vitae to jabfm@ med.wayne.edu or fax it to 313-577-9828. Thank you!

\section{Peer Reviewers}

Nahed Abdel-Haq

John Abramson

David A. Acosta

Alan M. Adelman

David C. Agerter

Erin J. Aiello

Stephen C. Alder

Grace A. Alfonsi

Mohammad M. Alkot

Deborah Allen*

William A. Alto*

Jennifer R. Amico

Christine Arenson

Bengt Arnetz

Vikram Arora

Latifi A. Asrar
Russell T. Attridge

John Bachman

Michael T. Baer

Seong-Yi Baik

Steffani R. Bailey

Suzanne Bakken*

Lodovico Balducci

Mikhail Bargan

Matthew G. Barnes*

Beth Barnet

Donald Barr

Kirsten Barrett

John W. Beasley

Bruce G. Bender

Ian M. Bennett*

Keisa Bennett
Terrell Benold

Alfred O. Berg*

George R. Bergus

Ravi Bhargava

Charles J. Billington

Juliann Binienda

Rajshekhar Bipeta

Arthur E. Blank

Debra Boardley

Thomas Bodenheimer

Linda Boise

John M. Boltri*

Robert C. Bowman

Massimo Broggini

Carlos Brotons

Subbaraju Budharaju 
Lucinda M. Buys

William Bynum

Thomas L. Campbell

John Cape

Roberto Cardarelli

Beth Careyva

Martha Carlough

Patricia A. Carney

A.P. Catinella

Andrew J. Cave

William E. Cayley, Jr.

Bruce A. Center

Bindiya Chauhan

Rajesh Chauhan

William E. Chavey

Frederick M. Chen

John A. Chiladakis

Kaj S. Christensen*

Lynn Clemow

Richard D. Clover

Andrew S. Coco

Andreas Cohrssen

Jeffrey S. Cook

Paul F. Crawford, III*

Jesse C. Crosson

Michael A. Crouch

Brian K. Crownover

Larry Culpepper*

Doyle M. Cummings

William Curry

Timothy P. Daaleman

Jessica Dalby

Steven D. Dallas

Jeanette M. Daly*

Peter G. Danis, III

Michael Z. David

Melinda M. Davis

Lauren DeAlleaume

Peter Del Fante

Jennifer E. DeVoe

L. Miriam Dickinson*

Joseph R. DiFranza

Sharon A. Dobie

Lisa Dodson

Stephen L. Doggett

Rowena J. Dolor*

Fanglong Dong

John N. Dorsch

Steven A. Dosh

Chyke A. Doubeni*

David Doukas
Marguerite Duane

Francis Dumler

Milton Eder

Marlene J. Egger

Thomas R. Egnew

Nancy C. Elder

William G. Elder, Jr.*

Gijs Elshout

John W. Ely

Shannon K. English

J.I. Escobar

Bernard G. Ewigman

Heather B. Fagan

Joshua J. Fenton*

Jeanne M. Ferrante*

Alexander G. Fiks

Kenneth S. Fink*

Kevin Fiscella

Colleen T. Fogarty

Patricia Fontaine

Chester H. Fox

Jennifer Frank

Andrea Franks

Brandi K. Freeman

Joshua Freeman

Allen Fremont

Sim S. Galazka

Ted Ganiats*

David Garr

Juan J. Gascon

Suzanne Gehl

Christa George

Cynthia M. Geppert

Jenenne Geske

James M. Gill

Dwenda K. Gjerdingen*

Rise B. Goldstein

Bharat Gopal

Laura M. Gottlieb

Edward Grandi

William B. Grant

Beverly B. Green*

Larry A. Green*

Lee A. Green*

Jessica L. Greenwood

Michelle Greiver

Michael Grover

Alexander B. Guirguis

Dave Hachey

Jacqueline R. Halladay

Margaret A. Handley
Diane M. Harper

Kelly Haskard Zolnierek

Robert L. Hatch*

Clare Hawkins

Ronni Hayon

Jennifer Hefner

Diana L. Heiman

John Heintzman*

Robin Helm

Vincent W. Hevern

John Hey, III

John M. Hickner

Paul C. Hicks

Michelle L. Hilaire

David E. Hildebrandt

Thomas C. Hines

Richard Hoffman

John Holmes

Christina T. Holt

Michael A. Horst

AmandaM.Howard-Thompson

Susan Hsieh

Shawna V. Hudson

Stephen W. Hwang

Jihad Irani

Henry R. Ivey, Jr.

Linda A. Jaber

Livia Jackson

Carlos Roberto Jaen

James Janisse

Muhammad A. Javed

Wiley D. Jenkins

Anthony F. Jerant

Xiangyang Jiao

Masahito Jimbo*

Mark S. Johnson*

Derek Jorgenson

Linda S. Kahn

Deepak Kamat

Rahul Kapur

Neil S. Kaye

Robert D. Keeley

Drew M. Keister

Omar A. Khan

Niharika Khanna

Kim S. Kimminau

Anne King

Dana E. King

Stella King*

Anita Y. Kinney

Benjamin E. Kligler 
Lyndee Knox

Richelle J. Koopman

Claudia A. Kozinetz

Martin Krepcho

N.R. Kressin

Alex H. Krist

Kurt Kroenke*

Robin L. Kruse

Anton J. Kuzel*

Sandi J. Lane

Carol J. Lange

Aaron M. Lear

Michael LeFevre

Patricia M. Lenahan

Sarah Lesko

Lenard I. Lesser

Lawrence Leung*

Winston Liaw

Andre F. Lijoi

Sonja M. Likumahuwa

Steven Y. Lin

Deerajnath Lingutla

Paula Darby Lipman

Charles F.S. Locke

Maja-Lisa Loechen

Everett E. Logue

David P. Losh

Margaret M. Love

Catherine L. Lysack

Michael K. Magill*

Arch G. Mainous, III

William T. Manard

Todd R. Marcy

Cara Marshall

Jeffrey Martin

Luca Mascitelli

Douglas M. Maurer

Catherine McCarthy

John McCarthy

Lisa McCarthy

M. Diane McKee

Michael M. McKee

David R. Mehr

Amitkumar Mehta

Virginia Miller

Nia S. Mitchell

James W. Mold*

Lynda Montgomery

Martina Mookadam

Motomi Mori

Christopher P. Morley*
Karie A. Morrical-Kline

Pierre A. Morris

Karen Muchowski

William J. Murdoch

Maureen A. Murtaugh

Zsolt J. Nagykaldi*

Mohammad Obadah Nakawah

Priya Nambisan*

Jatinder Narang

Susan Nash

Laeth S. Nasir

Jon O. Neher

Lynne S. Nemeth*

Robert J. Newman

Warren P. Newton

James Nicholson*

Polly H. Noel

Thomas E. Norris

Mary Patricia Nowalk

Jason O'Grady

Ann S. O'Malley

Thomas R. O'Neill*

M. Norm Oliver*

Mariusz Olszewski

Steven M. Ornstein

Wilson D. Pace*

J.F. Pagel

Scott A. Paluska

Nancy Pandhi*

Michael L. Parchman

Bennett Parnes

Himanshu Pathak

Donald Pathman*

Valory N. Pavlik

William S. Pearson

Deborah Peikes

Eribeth K. Penaranda

Kevin A. Peterson

Lars Peterson*

Margaret B. Planta

James D. Plumb

Charles D. Ponte

Shailendra Prasad

P. Prasadarao

Perry A. Pugno

Jon Puro

Mary Puttmann-Kostecka

Howard K. Rabinowitz

Mithun S. Rajput

Ravishankar Ramaswamy

Stephen Ratcliffe
Michael D. Reis

Robert L. Rhyne

Thomas B. Richards

Bradley A. Richie

Mark D. Robinson

Leonardo Roever

John C. Rogers

Thomas C. Rosenthal

Kenneth D. Royal

Robert S. Rudin

Mack T. Ruffin, IV

Terry S. Ruhl

Saria C. Saccocio*

Puneet Sahota

Amanda H. Salanitro

Ricardo Salazar

Daniel A. Salmon

Brooke E. Salzman

Enrique Sanchez Valdivieso

Paulo A. Santos

David Satin

Judith A. Savageau

Amir M. Sayem

Kenneth G. Schellhase

Joseph E. Scherger*

Eugene P. Schoener

Delia Scholes

Sarah H. Scholle

Sarina Schrager

Richard J. Schuster

Gerry K. Schwalfenberg

Kendra Schwartz*

Timothy H. Self

Peter A. Selwyn

Nicolas Senn

Richard K. Severson

Mohammed Shaik

Rajesh Sharma

Eric K. Shaw

Avani Sheth*

Stephanie L. Shimada

Anne R. Simpson

Vijay Singh

Peter C. Smith

E.J. Sondik

Hassan Soubhi

William Spears

Stuart M. Speedie

John B. Standridge 


Joseph W. Stanfield
Ra Nae Stanton
Michael Stehney
Paul D. Stein
Jost Steinhaeuser
Nancy G. Stevens
Melissa Stiles
Mark L. Stovak
Henrik Stovring
George D. Strelioff
Denise K. Sur
Andrew L. Sussman
John E. Sutherland
Christine Sutine Chang
Paul R. Swank*
Hazel Tapp*
Peter G. Teichman
Chris M. Terpening
Jeffrey Tiemstra
Stefan Topolski
Dennis Tsilimingras
Janice Tsoh

Matthew E. Ulven
Carole C. Upshur
Anje C. Van Berckelaer
Daniel J. Van Durme*
Charles P. Vega
William B. Ventres
Daniel C. Vinson
Michael Von Korff
Eric Wall*
Lorraine S. Wallace
Anne D. Walling
Alison M. Walton
Gregg Warshaw
Richard R. Watkins
Barry D. Weiss
Verna L. Welch
Frances K. Wen
Monica L. Wendel
Andrea Wendling
James J. Werner
David R. West*
Randy K. Wexler

Brett White
Thad Wilkins
Joanne E. Wilkinson
Keoki Williams
Robert L. Williams
Paul S. Williamson
Calvin L. Wilson
Daniel K. Winstead
Jason P. Womack
W. Michael Woods
Susan Woolhouse
Imam M. Xierali*
Jinping Xu
Mark J. Yaffe
Joseph R. Yancey
Barbara P. Yawn*
Mark W. Yeazel
Linda K. Zittleman
Allan Zuckoff
Karen Zulkowski
Stephen J. Zyzanski*

*Top reviewers in 2014 (based on quantity and quality of peer reviews written). 